\title{
Unmet Needs in Diabetic Macular Edema
}

\author{
Jennifer I Lim, MD \\ Professor of Ophthalmology and Director of Retina Services, Department of Ophthalmology and Visual Sciences, University of Illinois at Chicago Eye and Ear Infirmary
}

\begin{abstract}
Purpose: The purpose of this article was to review current therapies for diabetic macular edema (DME) and to discuss current unmet needs in the treatment of DME. Methods: The peer-reviewed literature was searched for information about the results of clinical trials on the treatment of DME. The Internet, Retina Society meeting abstracts, and publications (both peer-reviewed and non-peer-reviewed) were searched for information about ongoing clinical trials on the treatment of DME. Results: Laser photocoagulation until recently was the only treatment with proven efficacy in phase III randomized clinical trials for DME. Although visual acuity can be stabilized with laser treatment, visual improvement is uncommon. Recently, a phase III clinical trial showed that intravitreal ranibizumab with or without prompt laser resulted in superior visual acuity results at one year compared with eyes treated with triamcinolone or laser alone. Sustained drug delivery systems, preventive therapies, and novel drugs that prevent foveal lipid, reverse ischemia, and increase blood flow to the ischemic retina are needed. Conclusions: Current drug development is addressing the unmet needs of improving visual acuity in eyes with DME. Research is needed to develop novel therapies for sustained drug delivery, prevention of macular edema, and prevention of complications related to chronic macular edema.
\end{abstract}

\section{Keywords}

Diabetic macular edema, drug delivery, anti-vascular endothelial growth factor (VEGF), steroids

Disclosure: Jennifer I Lim, MD is on the Speakers' Bureau and is a member of the Executive Committee of the Ranibizumab for Edema of the mAcula in Diabetes (READ-2) Study, which received research funds from Genentech, is on the Advisory Board of Allergan, and has received research funds for study from ICON Bioscience. Received: January 18, 2011 Accepted: February 23, 2011 Citation: US Ophthalmic Review, 2011;4(1):101-4 DOI: 10.17925/USOR.2011.04.01.101 Correspondence: Jennifer I Lim, MD, Professor of Ophthalmology, Director of Retina Services, Department of Ophthalmology and Visual Sciences, University of Illinois at Chicago Eye and Ear Infirmary, 1855 West Taylor Street, Mail Code 648, Chicago, IL 60612. E: jennylim@uic.edu

Diabetic macular edema (DME) is, unfortunately, a very common problem affecting millions of diabetes patients worldwide. The Wisconsin Epidemiologic Study of Diabetic Retinopathy (WESDR) estimated that the prevalence of DME in patients with at least 15 years' duration of diabetes was $20 \%$ in type 1 diabetes mellitus (DM) patients, $25 \%$ in type 2 DM patients on insulin, and 14\% in type 2 DM patients not on insulin. ${ }^{1}$ In untreated DME, eyes will usually suffer significant visual Ioss. ${ }^{2}$ Fortunately, focal laser treatment for DME can prevent further visual loss in most eyes. Focal laser treatment was proven effective in the Early Treatment of Diabetic Retinopathy Study (ETDRS) and was hailed as a major breakthrough twenty years ago. ${ }^{1}$

In the ETDRS, 33\% of untreated eyes with foveal involvement suffered three or more lines of vision loss (moderate vision loss). ${ }^{2}$ In contrast, only $12 \%$ of eyes treated with focal laser photocoagulation suffered moderate visual acuity loss. ${ }^{2}$ However, lack of improvement in visual acuity following focal laser treatment is a major deficiency of this form of therapy. In fact, in the ETDRS only $11 \%$ of treated eyes gained 15 or more letters of visual acuity at year one and $16 \%$ of treated eyes gained 15 or more letters at year three. ${ }^{3}$ Recently, the Diabetic Retinopathy Clinical Research (DRCR) Network study used a modified ETDRS laser technique. Even with this technique, visual acuity improvement was not the norm. In eyes where visual acuity was $20 / 40$ or worse at baseline, the DRCR study found that
$29 \%$ of eyes gained 15 or more letters at one year and $6 \%$ of eyes lost 15 or more letters. ${ }^{4}$ Although a greater proportion of eyes gained visual acuity using the modified ETDRS technique at one year, three year data are not available and better treatment options that can result in overall improvement in visual acuity would be welcome in diabetes patients.

In addition to the absence of a reliable treatment that improves visual acuity in the majority of patients, there are several areas of unmet needs in the management of DME, including the need for sustained drug delivery systems, preventive therapies, and the ability to predict the development of DME. The unmet drug treatment needs include drugs that could perhaps prevent foveal lipid deposition, treat or reverse the effects of ischemia, and reverse chronic macular edema changes. Better drug delivery systems, such as intraocular implants and bioerodable implants, are required to achieve sustained drug delivery. Perhaps even more basic unmet needs are those for preventive therapies and the ability to predict which patients will develop macular edema based on genetic risk factors. Fortunately, there are several areas of active research focusing upon novel drugs (phase I and II studies), as well as improved and sustained delivery of previously studied drugs.

First, the greatest unmet need in DME is for a treatment that will improve visual acuity in the majority of patients. The era of anti-vascular 
endothelial growth factor (VEGF) therapy for age-related macular degeneration (AMD) ushered in pharmacologic treatments that actually improve visual acuity in about one-third to $40 \%$ of AMD eyes with subfoveal choroidal neovascularization (CNV) ${ }^{5,6}$ Previously, physicians could only hope to stabilize vision in these eyes. These treatments are currently undergoing clinical trials for the treatment of DME and proliferative diabetic retinopathy. The use of anti-VEGF treatments in diabetic retinopathy is based upon the well-demonstrated presence of elevated VEGF concentrations in eyes with proliferative diabetic retinopathy and DME. ${ }^{7.8}$

The first anti-VEGF drug studied in clinical trials for treatment of macular edema was pegaptanib sodium (Macugen ${ }^{\circledR}$, Eyetech Pharmaceuticals/ Novartis). Although intraocular injections of pegaptanib sodium given every six weeks for up to six months did result in decreased ocular coherence tomography (OCT) thickness as compared with that in sham-treated eyes, there was not a large change in visual acuity in treated versus sham eyes. Visual acuity improved by three or more lines in $18 \%$ versus $7 \%$ in control eyes $(p=0.12)$ at year one. ${ }^{9}$ The difference at three years is not yet published. The need for laser photocoagulation (between weeks 12 and 36 in the study) was much less in treated eyes than control eyes ( $0.3 \mathrm{mg}$ versus sham, $25 \%$ versus $48 \%$; $p=0.04$ ).

Recently, bevacizumab (Avastin ${ }^{\circledR}$, Genentech, South San Francisco, CA), as well as ranibizumab (Lucentis ${ }^{\circledR}$, Genentech, South San Francisco, CA) have been used in limited case series for treatment of DME. ${ }^{10-15}$ Ranibizumab and bevacizumab have been shown to rapidly decrease macular edema and thus normalize the ultrastructure of the macula in these diabetic eyes. In these case series, these anatomic changes seen on OCT have been associated with improved visual acuity. ${ }^{10-15}$

In the DRCR Network phase II study, 121 subjects were randomized to one of three groups: laser versus bevacizumab (two doses) versus bevacizumab with laser. There was evidence of reduced macular thickness in some eyes. In a study by Chun et al., 10 patients with centerinvolving macular edema underwent three intravitreal ranibizumab injections (baseline, month one, and month two). ${ }^{14}$ At six months, both the low dose $(0.3 \mathrm{mg})$ and high dose $(0.5 \mathrm{mg})$ groups experienced significant decreases in central OCT thickness compared with baseline. ${ }^{14}$

There are on-going studies comparing intravitreal anti-VEGF and other therapies both with each other and with standard laser photocoagulation. There are also studies looking at combination therapies (laser with adjunctive intravitreal anti-VEGF or steroid injections) compared with laser alone or anti-VEGF treatments alone. Some of these treatments have recently shown greater improvements in visual acuity outcomes compared with the ETDRS laser photocoagulation results.

The Ranibizumab for Edema of the Macula in Diabetes-1 (READ-1) study showed evidence of resolution of macular edema and improvement of visual acuity in treated eyes. At the primary end-point at month seven, median and mean OCT thicknesses were reduced by 261 and $246 \mu \mathrm{m}$ respectively from baseline-an $85 \%$ reduction in excess foveal thickness. ${ }^{15}$ Based on the positive results seen with READ-1, READ-2 was initiated. In READ-2, 126 eyes were randomized to ranibizumab versus ranibizumab with laser versus laser alone. The six-month primary outcome results from the READ-2 study have recently been evaluated. ${ }^{16}$ At six months, about one-quarter of patients gained three or more lines in the ranibizumab group versus $12 \%$ in the ranibizumab with laser group versus no eyes in the laser alone group. Two-year data are now available. After the initial six months, all three groups of patients were eligible to receive intravitreal ranibixumab every two months. At two years, there was no significant difference in the visual acuity outcomes, which were a gain of 7.7 (ranibizumab group initially), 5.1 (laser group initially), and 6.8 (combination group initially) letters. However, there were fewer injections required in year two for those eyes that received some laser treatment. Therefore, it appears that there is long-term benefit for the use of ranibizumab for DME. ${ }^{17}$

More recently, a DRCR study of 854 DME eyes has shown that intravitreal injections of ranibizumab with either prompt (three to 10 days) laser or deferred (24 weeks) laser resulted in superior visual acuity results at 12 months compared with intravitreal triamcinolone with laser or laser with sham injections. At one year, the mean change $( \pm$ standard deviation [SD]) in the visual acuity letter score from baseline was significantly greater in both the ranibizumab with prompt laser group $(+8 \pm 11$ letters; $\mathrm{p}<0.001)$ and the ranibizumab with deferred laser group $(+9 \pm 12$ letters; $p<0.001)$, but not in the triamcinolone with laser group $(+4 \pm 13$ letters; $p<0.31)$, compared with the sham-injection-plus-promptlaser group $(+3 \pm 13$ letters). In fact $28-30 \%$ of the ranibizumab group eyes gained $\geq 15$ letters from baseline at one year versus $15 \%$ for the laser with sham injection eyes. Therefore, we are finally seeing a treatment that can improve visual acuity in DME eyes. ${ }^{18}$

A clinical trial investigating the efficacy of ranibizumab as compared with controls (RESOLVE) is also under way. Phase III clinical trials are ongoing: A Study of Ranibizumab Injection in Subjects with Clinically Significant Macular Edema With Center Involvement Secondary to Diabetes Mellitus (RIDE); A Study of Ranibizumab Injection in Subjects With Clinically Significant Macular Edema With Center Involvement Secondary to Diabetes Mellitus (RISE); and DRCR Protocol I.

Apart from anti-VEGF antibodies, there are other drugs which inhibit VEGF, such as small interfering RNA (SiRNA). ${ }^{19}$ Use of an siRNA molecule which prevents the transcription of messenger RNA and thus prevents the formation of VEGF is another approach. Such a molecule, Cand5 (bevasiranib, OPKO Health, Miami, FL), was used in a phase II study, the RNAi Assessment of Bevasiranib in Diabetic Macular Edema (RACE) trial. This pilot phase II study enrolled 48 patients with DME in a double-masked, randomized trial. There was a trend towards decreased macular thickness between eight and 12 weeks. Further work is needed before this drug is accepted for DME.

There is also a VEGF Trap molecule that inhibits all isoforms of VEGF-A, other VEGF subtypes, such as VEGF-B and -C, and placental growth factor. ${ }^{20}$ Studies using these molecules are ongoing in eyes with DME. CLEAR-DME is a phase II study investigating the safety and efficacy of VEGF Trap in DME eyes. Recently, six-month and one-year positive results have been presented at meetings.

Corticosteroids, such as triamcinolone, have been used in the treatment of DME. ${ }^{21,22}$ The use of corticosteroids in the treatment of 
DME is based upon their ability to reduce VEGF-induced leakage in animal models. ${ }^{23}$ In a prospective study by Jonas et al., $25 \mathrm{mg}$ intravitreal triamcinolone improved treated eyes versus control eyes, but there was elevation of intraocular pressure (IOP) as a side effect. ${ }^{21}$ In a prospective, double-masked, placebo-controlled study by Gillies et al., $4 \mathrm{mg}$ intravitreal triamcinolone was compared with subconjunctival saline. Eyes treated with triamcinolone experienced an improvement in visual acuity (56\% treated versus $26 \%$ placebo had improvement of $>5$ letters logarithm of minimum angle of resolution [logMAR] visual acuity) and IOP elevation (68\% versus $10 \%$ increased by $>5 \mathrm{mmHg}$ ) compared with placebo eyes. ${ }^{22}$ The development of a steroid without the IOPelevating side effects would be welcome.

Other steroids, such as dexamethasone and fluocinolone, have been investigated in DME eyes. These drugs are available as the dexamethasone implant Posurdex ${ }^{\circledR}$ (Allergan, Irvine, CA) and the sustained-release fluocinolone implant Retisert ${ }^{\circledR}$ (Bausch and Lomb, Rochester, NY). The fluocinolone implant was studied in diabetes patients in the Fluocinolone Acetonide Implant Study (FAIS). ${ }^{24}$ In FAIS, visual acuity improved by three or more lines in $28 \%$ of implant-treated eyes versus $15 \%$ of standard of care eyes; edema resolved in $58 \%$ of implant-treated eyes versus $30 \%$ of standard of care eyes. ${ }^{24}$ However, there were significant adverse events: 95\% required cataract extraction and 30\% required glaucoma surgery. The Posurdex implant is currently being evaluated in a phase III clinical trial for treatment of macular edema.

Another class of drugs acts upon cell adhesion molecules, which are key mediators of inflammatory processes and play a role in the pathogenesis of diabetic retinopathy. ${ }^{25}$ Efalizumab (Genentech, South San Francisco, CA) inhibits the binding of LFA-1 (leukocyte functionassociated antigen-1) to intercellular adhesion molecule-1 (ICAM-1), ${ }^{26}$ and therefore inhibits adhesion of leukocytes to other cell types. In the Combined Approach to Treatment Using Ranibizumab and Efalizumab for Diabetic Macular Edema Study: The CAPTURE DME Study, the safety and tolerability of efalizumab, administered subcutaneously as a weekly dose $(1 \mathrm{mg} / \mathrm{kg})$, is compared with controls and with combination therapy with ranibizumab intravitreally.

With the potential promise of increased visual acuity, there is a need for sustained-release drug delivery devices. Durable treatments would be welcomed if studies show a need for continued monthly or other frequent injection regimens with the new drugs. Although there are clinical trials investigating sustained-release triamcinolone, devices for anti-VEGF drugs are needed. As mentioned above, there are currently sustained-release devices that deliver steroids. Perhaps this drug platform could also be utilized to deliver other drugs.

Another major unmet area of therapy is that for eyes with subfoveal deposits. At this time, there are no effective treatments for diabetic eyes with significant subfoveal lipid deposits. Following successful resolution of macular edema, foveal lipid can develop and limit visual acuity. In patients with chronic edema, foveal lipid also limits visual outcomes. Innovative treatments that could dissolve foveal lipid would be useful. Some studies have shown that surgery can remove the lipid but that visual acuity is limited..$^{27,28}$ Over a mean post-operative observation period of 43.2 months, visual acuity, which was improved in seven eyes
(54\%) at one year, was improved in only five eyes (38\%) compared with pre-operative visual acuity. The mean final visual acuity change was not statistically significantly improved in the treatment versus the control group. Many eyes developed atrophy after removal of the submacular hard exudates and macular edema.

A treatment to also prevent lipid deposition in eyes at higher risk of foveal lipid development would be useful. Whether statins, such as atorvastatin, can lead to reduction of hard exudates in DME eyes is controversial. ${ }^{29}$

To date, there is no drug that can increase blood flow significantly enough to relieve ischemia in the retina. Perhaps treatments to improve oxygen delivery or to cause reversal of hypoxia could be developed. Currently, there is an on-going clinical trial using an siRNA inhibitor of hypoxia-inducible factor-1 alpha (HIF-1). ${ }^{30} \mathrm{~A}$ pilot study has shown that HIF-1 is present in human proliferative diabetic membranes..$^{30}$ Rapamycin (Sirolimus, MacuSight) is currently being evaluated in a phase II study as a subconjunctival injection versus placebo control for eyes with DME. ${ }^{31}$ Rapamycin has immunosuppressive, antiangiogenic, antimigratory, antiproliferative, antifibrotic, and anti-permeability activity, as well as interrupting the mammalian target of rapamycin (mTOR) pathway (HIF-1 alpha pathway). A phase I study has shown rapamycin was safe and well-tolerated in all doses tested via two different routes of administration. Improvements in visual acuity and decreased foveal thickness by OCT were found for up to 180 days following a single administration of rapamycin.

Recently, there has been discussion of neural enhancement in the area of DME. Studies conducted with brimonidine in animals have shown increased responses from retinal ganglion cells in eyes with optic neuropathy and glaucomatous optic neuropathy. ${ }^{32}$ Studies in humans are in their early stages. Perhaps applications to DME will follow.

Chronic macular edema unresponsive to treatment is a significant problem. Various studies already mentioned have focused on this area. Perhaps combinations of therapy may be useful. Use of neuroprotective agents to protect the retinal photoreceptors and ganglion cell layers in eyes with long-standing edema may be helpful, while treatments to reduce the edema are given.

Lastly, effective preventive therapies for DME would be a welcome addition to our treatment armamentarium. The Age-related Eye Disease Study (AREDS) showed the efficacy of antioxidants and zinc in preventing the progression of AMD. ${ }^{33}$ Perhaps drugs to reverse hypoxia, or to limit its effects, would be useful in patients with diabetes. The ruboxistaurin study attempted to prevent retinopathy. ${ }^{34,35}$ The results were not significant for the prevention of macular edema in the vast majority of patients. However, the Protein Kinase C Diabetic Retinopathy Study 2 (PKC-DRS2) reduced the rate of sustained moderate visual from $9 \%$ to $5.5 \%$ over three years versus placebo. Patients had a $45 \%$ reduction in the risk of development of clinically significant DME and had a $26 \%$ reduction in their need for focal laser.

It is well accepted that improved systemic management delays the progression of retinopathy. The Diabetes Control and Complications Trial (DCCT), ${ }^{36}$ the UK Prospective Diabetes Study (UKPDS), ${ }^{37}$ and the 
Epidemiology of Diabetes Interventions and Complications Study (EDIC) ${ }^{38}$ showed that intensive glycemic control and blood pressure control delayed the onset of macular edema as compared with conventional treatment. Intensive glucose control had a sustained effect, with a 58\% risk reduction in the development of DME in the DCCT patients. Therefore improved systemic management of diabetes will likely also have a preventive effect on the development of DME.

The future may include research to investigate the genetics of DME. The identification of patients who are susceptible to macular edema may enable more preventive therapies to that population. Identification of genetic variants in the complement system has been possible in AMD. Similar work in macular edema may be useful.

Thus, there are several research pathways to pursue that may yield improvements in our ability to manage DME and, perhaps more importantly, prevent its development. I am hopeful that scientists and drug companies will continue to focus upon some of these pathways and that some of these efforts will yield a fruitful harvest of new treatments for DME.
1. Klein R, Klein B, Moss S, et al., The Wisconsin Epidemiologic Study of Diabetic Retinopathy, IV: diabetic macular edema Ophthalmology, 1984;91:1464-74.

2. Early Treatment Diabetic Retinopathy Study Research Group, Photocoagulation for diabetic macular edema: ETDRS report number 4, Int Ophthalmol Clin, 1987:27:265-72.

3. Early Treatment Diabetic Retinopathy Study Research Group, Photocoagulation for diabetic macular edema. Early treatmen diabetic retinopathy study report number 1, Arch Ophthalmol, 1985;103:1796-1806.

4. Diabetic Retinopathy Clinical Research Network, Comparison of modified-ETDRS and mild macular grid laser photocoagulation strategies for diabetic macular edema, Arch Ophthalmol 2007;125:469-80

5. Rosenfeld P, Brown DM, Heier J, et al., Ranibizumab for neovascular age-related macular degeneration, N Eng/ J Med, 2006:355:1419-31

6. Brown DM, Kaiser PK, Michels M, et al., Ranibizumab versus verteporfin for Neovascular age-related macular degeneration N Engl J Med, 2006;355:1432-44.

7. Aiello LP, Avery RL, Arrigg PG, et al., Vascular endothelial growth factor in ocular fluid of patients with diabetic retinopathy and other retinal disorders, N Eng/ I Med, 1994;331:1480-7.

8. Funatsu $\mathrm{H}$, Yamashita $\mathrm{H}$, Noma $\mathrm{H}$, et al. Increased levels of vascular endothelial growth factor and interleukin-6 in the aqueous humor of diabetics with macular edema, Am J Ophthalmol, 2002;133:70-7.

9. Macugen Diabetic Retinopathy Study Group, A phase randomized double-masked trial of pegaptanib, an antivascular endothelial growth factor aptamer, for diabetic macular edema, Ophthalmology, 2005;112:1747-57.

10. Diabetic Retinopathy Clinical Research Network, Scott IU Edwards AR, et al., A phase II randomized clinical trial of intravitreal bevacizumab for diabetic macular edema Ophthalmology, 2007:114:1860-7.

11. Ahmadieh $\mathrm{H}$, Ramezani $\mathrm{A}$, Shoeibi $\mathrm{N}$, et al., Intravitreal bevacizumab with or without triamcinolone for refractory diabetic macular edema; a placebo-controlled, randomized clinical trial, Graefes Arch Clin Exp Ophthalmol, 2008;246:483-9.

12. Shimura M, Nakazawa T, Yasuda K, et al., Comparative therapy evaluation of intravitreal bevacizumab and triamcinolone acetonide on persistent diffuse diabetic macular edema, Am I Ophthalmol, 2008:145(5):854-61.

13. Wu L, Martínez-Castellanos MA, Quiroz-Mercado $H$, et al., Twelve-month safety of intravitreal injections of bevacizumab (Avastin): results of the Pan-American Collaborative Retina Study Group (PACORES), Graefes Arch Clin Exp Ophthalmol, 2008;246(1):81-7

14. Chun DW, Heier JS, Topping TM, et al., A pilot study of multiple intravitreal injections of ranibizumab in patients with center involving clinically significant diabetic macular edema, Ophthalmology, 2006;113:1706-12.

15. Nguyen QD, Tatlipinar S, Shah SM, et al., Vascular endothelial growth factor is a critical stimulus for diabetic macular edema Am I Ophthalmol, 2006;142:961-9.

16. Do DV, Nguyen QD, Heier JS, et al., Six-month results of the READ-2 Study: Ranibizumab for edema of the macula in diabetes. A phase II clinical trial. Presented at American Society of Retinal Specialists Annual Meeting, October 13, 2008, Maui, HI.

17. Nguyen QD, Shah SM, Heier J, et al; READ-2 Study Group, Twoyear outcomes of the Ranibizumab for Edema of the mAcula in Diabetes (READ-2) Study, Ophthalmology, 2010;117(11):2146-51.

18. Elman MJ, Aiello LP, Beck RW, et al., Randomized trial evaluating ranibizumab plus prompt or deferred laser or triamcinolone plus prompt laser for diabetic macular edema, Ophthalmology, 2010:117(6):1064-77.

19. Singerman $L J$, Reich $S$, O'Shaughnessy $D$, et al., Bevasiranib for treatment of age-related macular degeneration and diabetic macular edema. Presented at Retina Society 40th Annual Scientific Meeting, September 28, 2007, Boston, MA.

20. Nguyen QD, Shah SM, Hafiz G, et al., A phase I trial of an IV-administered vascular endothelial growth factor trap for treatment in patients with choroidal neovascularization due to age-related macular degeneration, Ophthalmology, 2006;113:1522-32.

21. Jonas JB, Kreissig I, Sofker A, Degenring RF, Intravitreal injection of triamcinolone for diffuse macular edema, Arch Ophthalmol, 2003:121:57-61.

22. Gillies MC, Sutter FK, Simpson JM, et al., Intravitreal triamcinolone for refractory diabetic macular edema: two-year results of a double-masked, placebo-controlled, randomized clinical trial, Ophthalmology, 2006;113:1533-8.

23. Zhang $\mathrm{X}, \mathrm{Bao} \mathrm{S}$, Lai $\mathrm{D}$, et al., Intravitreal triamcinolone acetonide inhibits breakdown of the blood-retinal barrie through differential regulation of VEGF-A and its receptors in early diabetic rat retinas, Diabetes, 2008:57:1026-33.

24. Ip MS, Comstock T, Fluocinolone acetonide intravitreal implant to treat diabetic macular edema: 3-year results of a multicenter clinical trial. Presented at Retina Society/Club Jules Gonin Annual Meeting, Oct 15-20, 2006, Cape Town, South Africa.

25. Adamiec-Mroczek J, Oficjalska-Mlynczak J, Assessment of selected adhesion molecule and proinflammatory cytokine levels in the vitreous body of patients with type 2 diabetes role of the inflammatory-immune process in the pathogenesis of proliferative diabetic retinopathy, Graefes Arch Clin Exp Ophthalmol, 2008;246:1665-70.

26. Combined approach to Treatment Using Ranibizumab and Efalizumab for Diabetic Macular Edema Study: The CAPTURE
DME Study. Available at: www.clinicaltrials.gov (accessed August 28,2008 ).

27. Takaya $\mathrm{K}$, Suzuki $\mathrm{Y}$, Mizutani $\mathrm{H}$, et al., Long-term results of vitrectomy for removal of submacular hard exudates in patients with diabetic maculopathy, Retina, 2004:24:23-9.

28. Sakuraba T, Suzuki $Y$, Mizutani $H$, Nakazawa M, Visual improvement after removal of submacular exudates in patients with diabetic maculopathy, Ophthalmic Surg Lasers, 2000;31:287-91.

29. Gupta A, Gupta V, Thapar S, Bhansali A, Lipid-lowering drug atorvastatin as an adjunct in the management of diabetic macular edema, Am I Ophthalmol, 2004:137:675-82.

30. Lim JI, Spee C, Hinton D, Determination of HIF-1 in surgically excised preretinal membranes of diabetic patients compared to non-diabetic patients. Poster F-7. Presented at Retina Society 40th Annual Scientific Meeting, September 28, 2007, Boston, MA.

31. Blumenkranz MS, Dugel P, Solley WA, et al., Rapamycin (Sirolimus) for the treatment of diabetic macular edema: Preliminary results of a prospective phase 1 trial. Presented at Retina Society 40th Annual Scientific Meeting, September 28, 2007, Boston, MA.

32. Wheeler L, WoldeMussie $E$, Lai R, Role of alpha-2 agonists in neuroprotection, Surv Ophthalmol, 2003;48(Suppl. 1):S47-51.

33. Age-Related Eye Disease Study Research Group, A randomized, placebo-controlled, clinical trial of high-dose supplementation with vitamins $C$ and $E$, beta carotene, and zinc for age-related macular degeneration and vision loss: AREDS report no. 8, Arch Ophthalmol, 2001;119:1417-36.

34. PKC-DRS Study Group, The effect of ruboxistaurin on visual loss in patients with moderately severe to very severe nonproliferative diabetic retinopathy: initial results of the Protein Kinase $\mathrm{C}$ beta Inhibitor Diabetic Retinopathy Study (PKC-DRS) multicenter randomized clinical trial, Diabetes, 2005;54(7):2188-97.

35. The PKC-DRS Study group, Effect of ruboxistaurin on visua loss in patients with diabetic retinopathy, Ophthalmology, 2006;113:2221-30.

36. Diabetes Control and Complication Trial Research Group, The effect of intensive treatment of diabetes on the development and progression of long-term complications in insulindependent diabetes mellitus, N Eng/ J Med, 1993;329:977-86.

37. UK Prospective Diabetes Study Group, Intensive blood-glucose control with sulphonylureas or insulin compared with conventional treatment and risk of complications in patients with type 2 diabetes. UKPDS 33, Lancet, 1998;352:837-53.

38. The Diabetes Control and Complication Trial/Epidemiology of Diabetes Interventions and Complications Research Group, Retinopathy and nephropathy in patients with type 1 diabetes four years after a trial of intensive therapy, $N$ Engl I Med, 2000;342:381-9. 